\title{
GAUDÍ, CONSTRUCTOR (LA MATERIALIZACIÓN DE UNA ARQUITECTURA SINGULAR)
}

\author{
(GAUDI, CONSTRUCTOR (THE MATERIALIZATION OF A UNIQUE ARCHITECTURE)) \\ Antoni González Moreno-Navarro, Arquitecto.
}

\section{RESUMEN}

La aportación de Gaudi a la historia de la Arquitectura no se puede asociar directamente al cambio radical que la disciplina iba a sufrir en las primeras décadas de nuestro siglo. Gaudi no fue, en este sentido, un precursor, a pesar de que su influencia en la renovación formal de la arquitectura europea sea un hecho reconocido, sólo matizado por la valoración que cada uno hace de sus obras según la emoción que le producen. El acercamiento objetivo a su obra desde ópticas sectoriales, como es el caso de la construcción -facilitado ahora por las restauraciones en curso-, puede ser útil para analizar esa aportación siempre discutida.

Desde el punto de vista del autor del artículo, la auténtica aportación de Gaudi a la cultura arquitectónica europea es su obra en si misma, independientemente de su

trascendencia posterior; pero también su actitud ante la arquitectura: la pasión por imaginar pero también la pasión por construir, la voluntad de no disociar nunca los aspectos compositivos y formales de los genuinamente constructivos. Una lección útil en el momento confuso de la arquitectura que vivimos hoy.

\section{SUMMARY}

The contribution of Gaudi to the history of architecture cannot be associated directly with the radical change which the discipline was to undergo in the first decades of our century. Gaudi was not, then, a precursor, despite the fact that his influence in the formal renovation of European architecture is a recognized fact, qualifed only by the opinion one has of his works according to the emotional effect they produce. The objective approximation to his work from sectorial points of view, as in the case of the construction - now facilitated by the restoration underway - can help analyze this always controversial contribution.

From the point of view of the author of this article, the real contribution of Gaudi to European architectural culture is his work itself, independently of its later trascendence; the passion to imagine, but also the passion to build, the desire to never disassociate compositive and formal aspects from genuinely constructive ones, is a useful lesson at this confusing moment in architecture we are living today.
Se ha dicho demasiadas veces que Gaudí fue un revolucionario de la arquitectura. Tanta retórica han gastado algunos adeptos del arquitecto catalán al afirmarlo, que han provocado a menudo reacciones desmitificadoras, a veces tan pueriles como la causa que las genera.

Que la obra de Antoni Gaudí i Cornet (1852-1926) fue decisiva en la renovación formal de la arquitectura europea del siglo XIX, es un hecho tan evidente como reconocido. Otra cosa es la valoración que cada uno pueda hacer de sus propuestas formales, ya que nunca podremos substraernos del parámetro del "gusto", que no es otra cosa que la traducción en forma de jui. cio crítico de la emoción que nos produce una obra de arte.
Pero la emoción (de uno u otro signo), siempre subjetiva, no debería impedir el análisis objetivo de las demás presumibles aportaciones - genéricas o sectoriales - de una obra creativa como la de Gaudi. Es decir, la valoración de su aportación a la Arquitectura, más allá de las consideraciones formales, una aportación puesta en duda a menudo. No es tampoco fácil, ya que esa valoración vendrá siempre condicionada por el particular criterio que de la arquitectura tenga cada uno; de los aspectos de la arquitectura que cada uno reconoce como más significativos en función, muchas veces, de la propia especialidad.

Por ello, los esfuerzos por acercarse objētivamente a la obra gaudiniana desde ópticas sectoriales (el dise- 
ño, la construcción, el pensamiento), suponen un buen camino, especialmente si no nacen como consecuencia de ese vaivén, bien mitificador, bien desmitificador, al que algunos críticos y profesionales se creen obligados de vez en cuando.

Desde el punto de vista de su manera de entender la construcción, es decir, de cómo concibió Gaudí la materialización de su singular arquitectura, estamos en un buen momento para intentar un nuevo acercamiento. Lo facilita el conocimiento más profundo de su legado que nos permite la restauración que ahora se hace de casi todas sus obras. Con este trabajo - necesariamente parcial - referido a algunos aspectos de Gaudi constructor, queremos sumarnos a las aportaciones hechas en épocas recientes, aunque en circunstancias diversas, que han de colaborar a un juicio más desapasionado de la figura de Gaudí (1).

En el acercamiento crítico a Gaudí constructor, conviene comenzar por deshacer algunos equívocos. Conviene matizar, por citar unos ejemplos tópicos como el de que Gaudí fuera "el último gótico" o el introductor en la arquitectura moderna de la "planta libre"; opiniones en las que late una valoración de la arquitectura desde la concepción global estructural del edificio. Quizás una de las primeras conclusiones a las que nos llevan los estudios actuales derivados de las restauraciones en curso, es que, precisamente, la concepción de la estructura portante como realidad autónoma, no era uno de los objetivos prioritarios de Gaudí. Así lo podremos comprobar tanto en las obras de juventud -como el Palau Güell-, como en obras de madurez, representadas quizás mejor que por ninguna otra por la Cripta de la Colonia Güell, donde - como opinan José Luis González y Albert Casals - ya no cabe hablar de estructura independiente del edificio, pues allí, estructura y edificio son una misma cosa.

Gaudí tampoco fue un innovador en cuanto a los materiales de construcción. Como indica certeramente en su trabajo el profesor Bassegoda, Gaudí creó formas arquitectónicas realmente insospechadas, que asumían un trabajo mecánico de forma no habitual, y utilizó los materiales de forma singular e imaginativa, pero se trata siempre de los materiales tradicionales que él tan bien conocía. Fue, precisamente, esa utilización indiscriminada y a veces aleatoria, o sin suficientes garantías, de algunos materiales - a menudo al límite de sus posibilidades expresivas, pero también de su capacidad resistente - la que si bien permitió a Gaudí imaginar soluciones nuevas, también ha resultado la causa de algunos fracasos constructivos. La Casa Milà es un buen ejemplo, como pone de manifiesto el trabajo del Dr. Rafael Vila.

Los materiales y las soluciones constructivas, incluso la concepción del sistema estructural, no eran, pues,

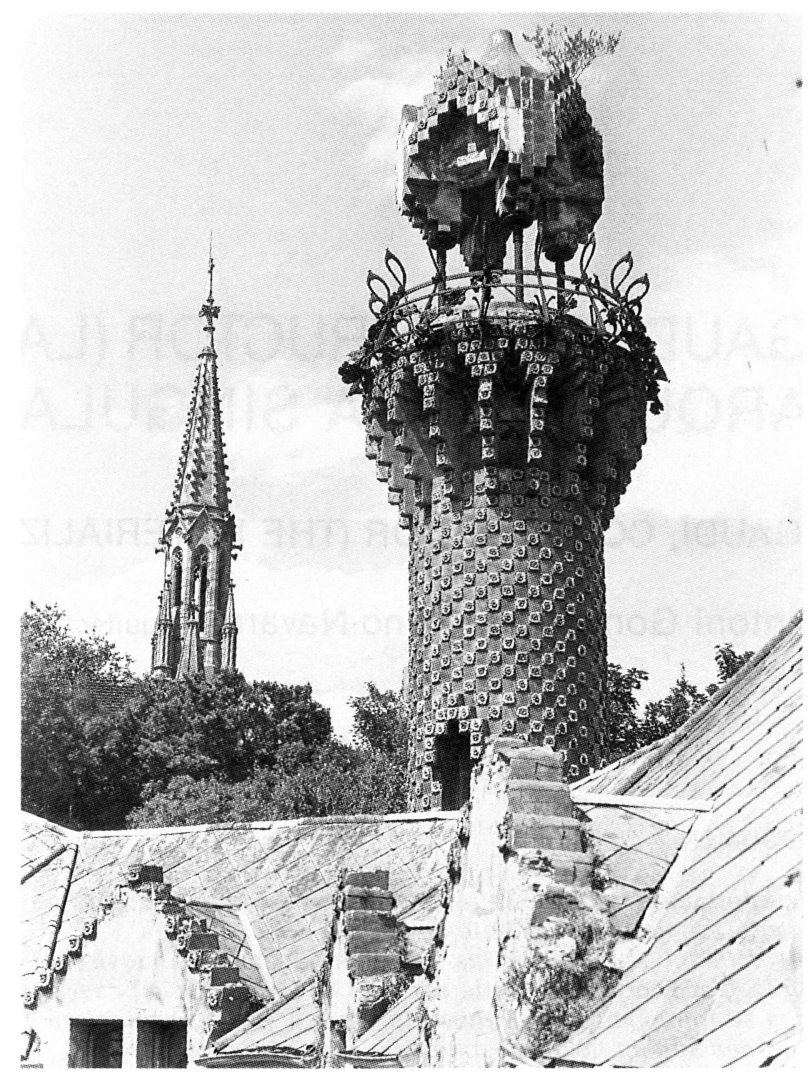

Fig. 1.- La renovación formal puesta de manifiesto en la torre del Capricho de Comillas (Cantabria), obra de Gaudi (1883-1885), frente a la torre de la capilla-panteón del marqués, obra de su maestro Joan Martorell (1878-1880). (Foto Arxiu GMN, agosto de 1971).

para Gaudí más que medios al servicio de la expresividad formal y espacial de su imaginativa arquitectura. ¿Quiere decir esto que Gaudí también contribuyó a crear la dicotomía aparente del artificioso binomio arquitectura-construcción, que tan penoso estigma ha dejado en gran parte de la arquitectura de nuestro siglo? ¿Quiere decir que definitivamente hay que encasillar a Gaudí como un renovador formal, un soñador de formas, que tuvo que utilizar triquiñuelas "poco arquitectónicas" para sostenerlas?

Si analizamos desapasionada pero pausadamente la obra de Gaudí desde la óptica de la construcción, comprobaremos cómo es de falaz afirmar que aquella arquitectura se concibió olvidándose que debía ser construida. Bien al contrario, detrás de cada forma nueva late una búsqueda inquieta de una solución constructiva. El hecho de que nunca los materiales utilizados fueran revolucionarios, o que las soluciones proyectadas no hayan supuesto el inicio de una nueva manera universal de construir, no puede minimizar la genialidad del Gaudí constructor, tan imaginativo, tan personal, tan apasionado como el Gaudí diseñador.

La aportación de Gaudí a la historia de la Arquitectura no se puede asociar directamente al cāmbio radical que la disciplina iba a sufrir en las primeras décadas de 


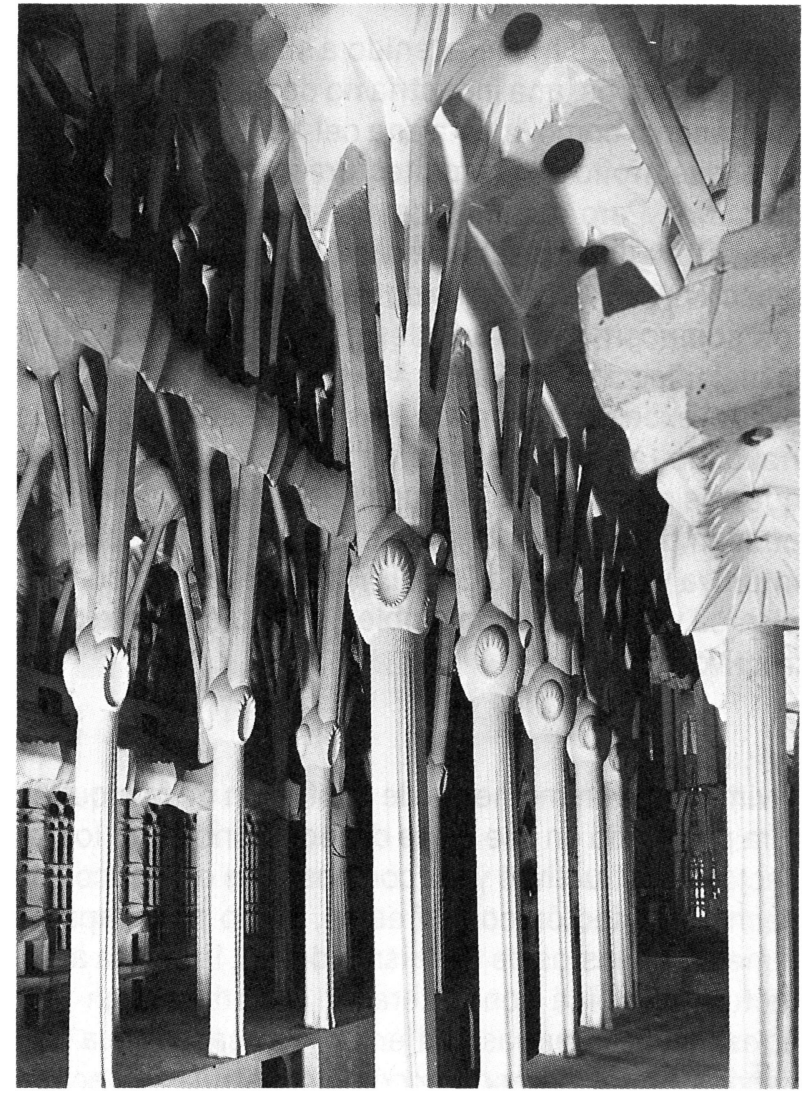

Fig. 2.-Modelos en yeso para la nave del templo de la Sagrada Familia de Barcelona. (Foto Arxiu Sagrada Familia). nuestro siglo (enterremos definitivamente el tópico de Gaudí precursor del Racionalismo). Que las concepciones formales y espaciales de algunos grandes maestros del siglo XX tienen sus raíces en la arquitectura de Gaudí, es evidente; pero no es suficiente para hacer del arquitecto catalán un precursor o un profeta.

Las auténticas aportaciones - desde mi punto de vista, naturalmente- de Gaudí a la historia de la Arquitectura son dos. En primer lugar, su obra en sí misma. (¿Por qué necesariamente ha de valorarse una obra creativa en función de una posible escuela o influencia universal posterior? El goce estético del paseo por la cubierta de la Pedrera o la atracción sugestiva del recorrido por el Park Güell - por citar dos ejemplosestán ahí, al alcance de cualquier espectador de cualquier época, sin que necesiten ser legitimadas por la influencia posterior de esas obras). Y en segundo lugar, la actitud de Gaudí ante la arquitectura. La actitud ante la recreación formal, ante la armonía urbana, ante la investigación constructiva; la pasión por imaginar pero también la pasión por construir.

Como dice en su artículo Josep Rovira Pey, refiriéndose al Park Güell, sorprende cómo con una tecnología tan primitiva fue capaz Gaudi de crear unas formas tan bellas. Sólo la imaginación y la pasión puesta por Gaudí en la construcción como elemento esencial del hecho arquitectónico, lo explica.

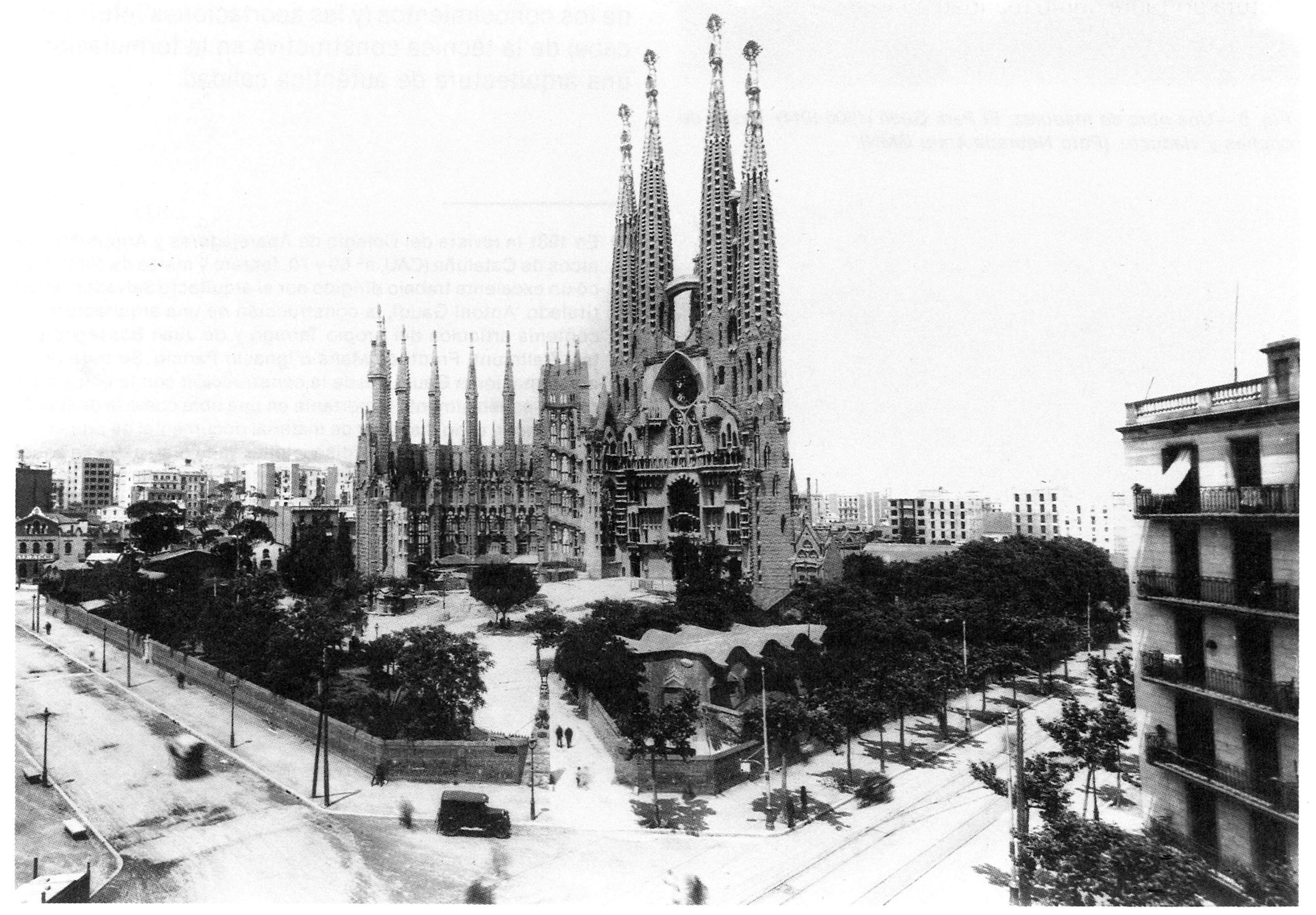

Fig. 3.-La Sagrada Familia en 1933. Las únicas obras del templo atribuibles a Gaudí. (Foto Arxiu Mas). 


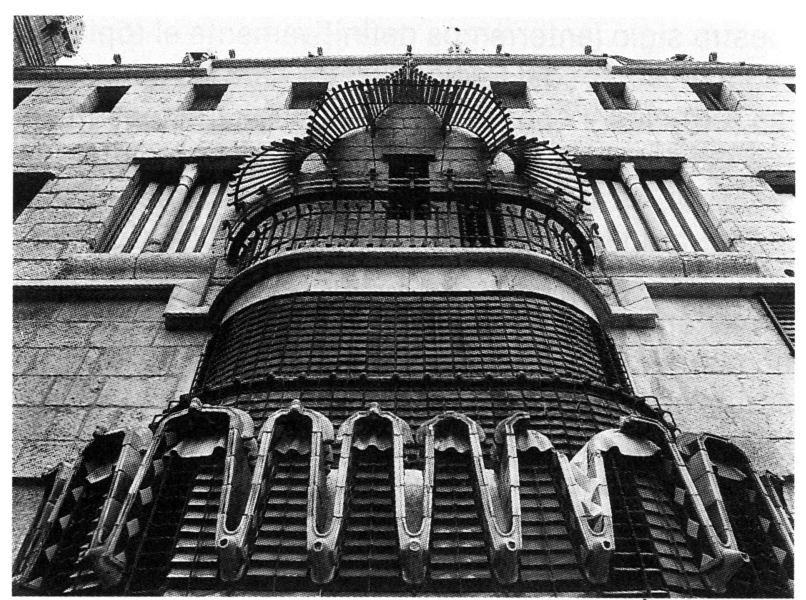

Fig. 4.-Una obra de juventud: El Palau Güell (1885-1890). Tribuna y umbráculo de la fachada de mediodia. (Foto Jordi Isern, marzo de 1990).

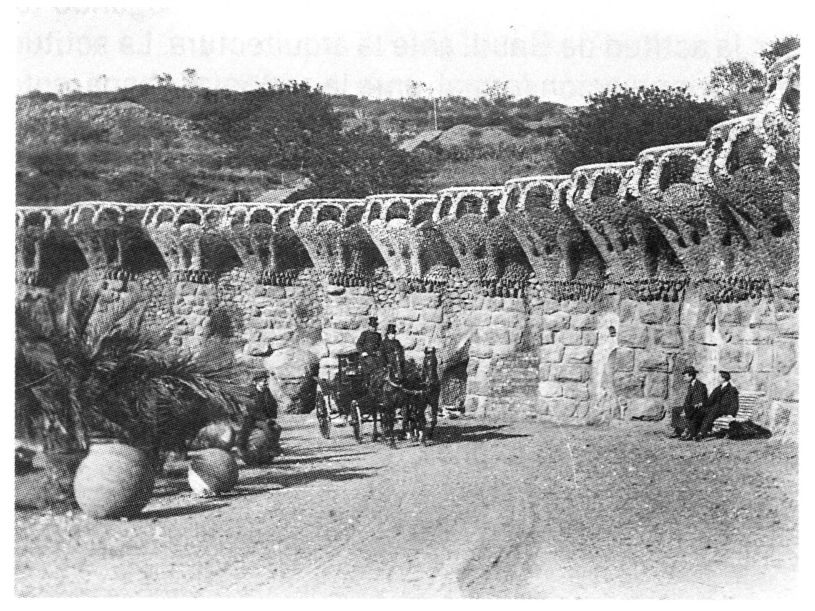

Fig. 5.-Una obra de madurez: El Park Güell (1900-1914). Paseo de coches y viaducto. (Foto Nebreda-Arxiu GMN).
Quizás si Gaudí hubiera tenido a su alcance unos materiales nuevos, una industria no de raíz profundamente artesanal como la catalana del XIX, quizás su papel en el desarrollo de la arquitectura moderna hubiera sido mayor. Pero qué más da. Esa pasión por imaginar y construir (imaginar la construcción, construir la imaginación) esa voluntad de no disociar nunca los aspectos compositivos y formales de los constructivos es la mejor lección de Gaudí. A pesar de los errores. A pesar del exceso de confianza en materiales que han defraudado las expectativas. La sorpresa, o los reparos que nos producen hoy algunas de esas soluciones, no pueden hacer variar la consideración de la capacidad intuitiva y creativa de Gaudí, y la racionalidad con que plantea y resuelve cada problema genuinamente constructivo.

Vivimos hoy un momento de confusión de la arquitectura plasmado en ese grave desequilibrio entre los aspectos constructivos y los compositivos o plásticos del hecho arquitectónico (ahí están, como polos aparentemente opuestos de un mismo drama, la bonita arquitectura olímpica con goteras y la hortera high tech artesanal de nuestras periferias). Revisar ahora a Gaudi constructor, comprobar cómo construía su peculiar arquitectura, aporta luz sobre la subsidiaridad relativa de esos aspectos (la construcción es un medio, no un fin) pero también sobre la trascendental importancia de los conocimientos (y las aportaciones intuitivas, si cabe) de la técnica constructiva en la formulación de una arquitectura de auténtica calidad.

(1) En 1981 la revista del Colegio de Aparejadores y Arquitectos técnicos de Cataluña (CAU, $n^{\circ} 69$ y 70 , febrero y marzo de 1981), publicó un excelente trabajo dirigido por el arquitecto Salvador Tarragó, titulado 'Antoni Gaudi, la construcción de una arquitectura', que contenia articulos del propio Tarragó y de Juan Bassegoda, Rafael Bellmunt, Fructuós Mañá e Ignacio Paricio. Se trata de una aproximación a Gaudi desde la construcción con la única limitación -especialmente importante en una obra como la de Gaudique supone el no disponer de material documental de primera mano y no haber podido aún "diseccionar" una gran parte de aquella arquitectura. La restauración que se hace ahora de muchas de las obras del arquitecto ha permitido mejorar el conocimiento de algunos pasajes constructivos de esa arquitectura. 